\title{
Má rotação intestinal na adolescência e suas consequências no diagnóstico tardio: relato de caso
}

Intestinal malrotation in adolescence and its consequences in late diagnosis: case report

Gislei Frota $\operatorname{Aragão}^{1}$ (D), Ronaldo Sobreira Guedes Filho ${ }^{1}$ (D), João Henrique Freitas Colares ${ }^{2}$ (D) Jamille Linhares Feijó $^{2}$ (D), Manuel Luiz Filgueira Oliveira ${ }^{3}$ (D) , Yasmin Ingrid Santos Oliveira $^{1}$ (D)

\begin{abstract}
RESUMO
A má rotação intestinal é uma anomalia congênita de causa desconhecida, sendo vômitos biliosos um dos diagnósticos diferenciais na infância, e em adultos consiste em um difícil diagnóstico. Adolescente de 17 anos iniciou um quadro de dor mesogástrica, de caráter intermitente, com piora pós-prandial. Apresentou dores abdominais intensas, vômitos biliosos, desidratação e desnutrição severa. Foram realizadas endoscopia e seriografia digestiva alta, que sugeriram má rotação intestinal. O paciente foi submetido ao procedimento de Ladd por videolaparoscopia. Durante a cirurgia, foi achado o ceco em hipocôndrio esquerdo, estômago e primeira porção duodenal dilatada por presença de banda de Ladd na segunda porção duodenal. Foi realizado lise das bandas de Ladd, apendicectomia e reposicionamento das alças. Recebeu alta hospitalar após quatro dias. A má rotação intestinal em adultos é de difícil identificação e o procedimento de Ladd por videolaparoscopia é a técnica cirúrgica de escolha para esses pacientes sem complicações agudas.
\end{abstract}

Palavras-chave: anormalidades congênitas; anormalidades do sistema digestório; intestinos; diagnóstico tardio; adolescente; laparoscopia.

\begin{abstract}
Intestinal malrotation is a congenital anomaly of unknown cause, being bilious vomiting one of the differential diagnoses in childhood, although its diagnosis in adults is difficult. A 17-year-old adolescent started with mesogastric pain, of intermittent character, with postprandialworsening. He presented severe abdominal pains, bilious vomiting, dehydration and severe malnutrition. He underwent endoscopy and upper digestive seriography, which suggested intestinal malrotation. The patient underwent the Ladd procedure by video laparoscopy. During the surgery, the cecum was found in the left upper quadrant, stomach and first duodenal portion dilated by presence of Ladd band in the second duodenal portion. Lysis of the bands of Ladd, appendectomy and repositioning of the handles were performed in the patient. He was discharged after four days. It is difficult to identify intestinal malrotation in adults, and the Ladd laparoscopic procedure is the surgical technique of choice for these patients without acute complications.

Keywords: congenital abnormalities; digestive system abnormalities; intestines; delayed diagnosis, adolescent; laparoscopy.
\end{abstract}

\section{INTRODUÇÃO}

A má rotação intestinal é uma anomalia congênita ocasionada por uma rotação incompleta ou não rotação do intestino embrionário no eixo da artéria mesentérica superior durante o desenvolvimento embriológico. Estudos apontam que $64 \%$ dos casos tornam-se evidentes, clinicamente, nos primeiros meses de vida e $82 \%$ até o primeiro ano. A incidência dessa malformação é ainda desconhecida, entretanto estimativas apontam uma variação entre 1:200 a 1:6 mil nascidos vivos. Em adultos, a incidência é de 0,2\%, porém por ser uma doença de maior prevalência pediátrica, o seu diagnóstico em adultos se torna difícil, aumentando o risco de desencadear consequências graves, tanto agudas como crônicas. ${ }^{1}$

\footnotetext{
${ }^{1}$ Universidade Estadual do Ceará - Fortaleza (CE), Brasil.

${ }^{2}$ Hospital Infantil Albert Sabin - Fortaleza (CE), Brasil.

${ }^{3}$ Escola de Saúde Pública do Ceará - Fortaleza (CE), Brasil.

Autor correspondente: Gislei Frota Aragão - Núcleo de Pesquisa e Desenvolvimento de Medicamentos, Universidade Federal do Ceará - Rua Coronel Nunes de Melo, 1.000 - Campus do Porangabussu - Rodolfo Teófilo - CEP: 60430-275 - Fortaleza (CE), Brasil - E-mail: frotaaragao@ hotmail.com

Recebido em 07/08/2017. Aceito para publicação em 31/03/2018.
} 


\section{RELATO DE CASO}

Paciente, masculino, 17 anos, refere dor epigástrica desde os 4 anos de idade, com início progressivo, irradiando para o dorso, com piora pós-prandial, ao acordar e em decúbito dorsal, associado a regurgitações, vômitos biliosos e eructações.

Procurou a emergência do hospital após intensificação das dores epigástricas e vômitos biliosos, além de apresentar-se havia 15 dias sem evacuar, com perda ponderal de $4 \mathrm{~kg}$ em uma semana e com má alimentação, por causa do quadro emético. Apresentava estado geral comprometido, fácies de dor, acianótico, afebril, anictérico, hipocorado, eupnêico, caquético, desidratado, orientado e consciente em tempo e espaço, murmúrio vesicular universal sem ruídos adventícios, ritmo cardíaco regular, em dois tempos, bulhas normofonéticas, sem sopro cardíaco, pulsos periféricos palpáveis e acianótico, com tempo de perfusão capilar menor do que 3 segundos. $\mathrm{O}$ abdome encontrava-se escavado, doloroso à palpação profunda, principalmente em região epigástrica, sem viceromegalias e com ruídos hidroaéreos presentes sem alterações.

$\mathrm{O}$ paciente foi submetido à endoscopia digestiva alta com biopsia do esôfago, estômago e duodeno, à ultrassonografia abdominal e à seriografia baritada de trânsito intestinal. $\mathrm{Na}$ endoscopia, foi diagnosticado esofagite erosiva grau $\mathrm{B}$ de Los Angeles, hérnia hiatal $(3 \mathrm{~cm}$ do pinçamento diafragmático), esôfago de Barrett e pangastrite enantematosa moderada. Resultados anatomopatológicos do esôfago e do duodeno dentro dos limites das normalidades; no estômago apresentou gastrite crônica leve em mucosa gástrica de padrão pilórico com pesquisa para Helicobacter pylori negativo. Na ultrassonografia abdominal, não foi evidenciado alterações. Na seriografia, demonstrou acentuada dilatação difusa do estômago e do duodeno até o ângulo de Treiz com retardo da passagem de contraste para alças jejunais, sugerindo processo semiobstrutivo, além de leve dilatação difusa de alças jejunoileais, com região ileocecal sem alterações (Figura 1).
O caso foi diagnosticado como semiobstrução intestinal por má rotação intestinal. Foi indicada videolaparoscopia com correção cirúrgica por meio do procedimento de Ladd e apendicectomia profilática. Durante a cirurgia, foi achado o ceco em hipocôndrio esquerdo, estômago e primeira porção duodenal dilatada por presença de banda de Ladd na segunda porção duodenal. Conduta intraoperatória: lise das bandas de Ladd, apendicectomia com eletrocoagulação do meso e ligadura com 2 clips proximais e 1 distal no apêndice, verificação das alças desde a válvula ileocecal até o ângulo de Treiz, sem evidências de alterações e reposicionamento das alças e ceco sem fixação.

No pós-operatório, paciente evoluiu bem, sem dor e vômitos. Após 8 meses da cirurgia, o paciente ganhou $13 \mathrm{~kg}$, porém com leves dores abdominais eventualmente. $\mathrm{O}$ paciente voltou a frequentar a escola, com melhora da concentração, da memória e do humor.

O presente estudo foi aprovado pela Comissão de Ética em Pesquisa do Hospital Albert Sabin (nº 51515115.8.0000.5042), onde fora realizado o trabalho, e está de acordo com os princípios éticos contidos na Declaração de Helsinki, 2013.

\section{DISCUSSÃO}

O paciente passou a maior parte da infância sintomático, manifestando sintomas dispépticos progressivos e, posteriormente, sinais de obstrução intestinal, provavelmente devido à obstrução pelas bandas de Ladd na segunda porção do duodeno, havendo alguns períodos de intensificação das dores e dos vômitos. Desencadeou ao longo dos anos um refluxo gastroesofágico com hérnia hiatal e, posteriormente, pangastrite enantematosa, esofagite erosiva grau B de Los Angeles e esôfago de Barrett. Realizamos uma pesquisa à procura da correlação de tais complicações com a má rotação intestinal no banco de dados do PubMed, entretanto nada foi encontrado, justificando a gravidade e a importância desse caso para aumentar o conhecimento desse assunto em adultos. Durante a cirurgia do paciente não foram vistos volvo, hérnia e alça isquêmica.
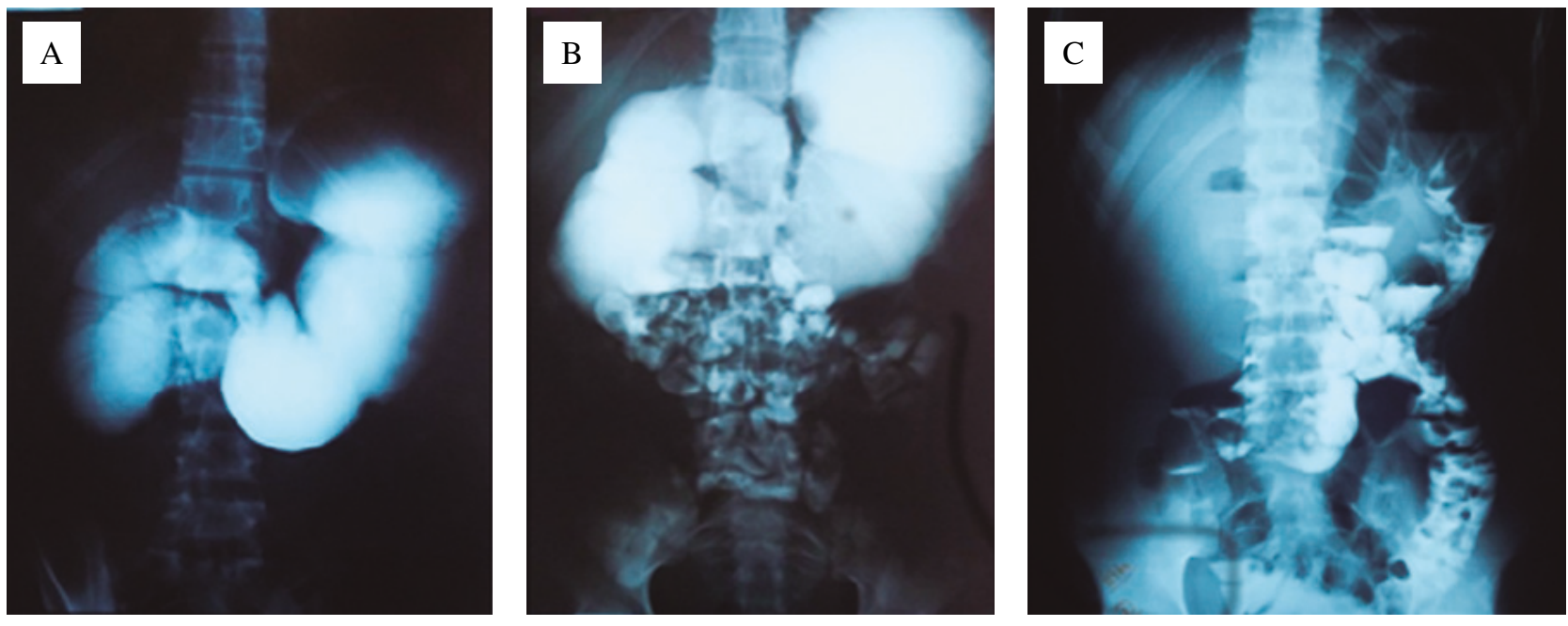

Figura 1. Aspectos da seriografia gastroduodenal e do trânsito intestinal: (A) dilatação gástrica e duodenal difusa; (B) retardo na passagem do contraste para o intestino delgado; $(\mathrm{C})$ variação de posição do cólon, predominando do lado esquerdo. 
Pelo padrão de vômitos biliosos de longa data e de dor abdominal após alimentação, associado ao quadro consumptivo, foi pensado em obstrução intestinal de via alta do trato gastrintestinal. Logo foi solicitada ultrassonografia, que não evidenciou nada por ser examinador-dependente. Por isso, foi solicitada uma seriografia gastroduodenal com trânsito intestinal, considerada por muitos autores como padrão-ouro para avaliar a má rotação intestinal. Nesse exame foram visualizados dilatação gástrica e duodenal difusa, retardo na passagem do contraste para o intestino delgado e variação de posição do cólon, predominando do lado esquerdo. Poderia também ser solicitada uma tomografia, considerada outro exame excelente para avaliar um quadro obstrutivo, podendo evidenciar relação inversa entre os vasos mesentéricos superiores (veia situada à esquerda da artéria), ausência ou hipoplasia do processo uncinado do pâncreas e alterações na disposição das alças e da transição duodenojejunal, situando-se à direita do abdome, conforme alguns autores citam em seus trabalhos. ${ }^{1-6}$

O procedimento de Ladd consiste, primeiramente, na resolução de eventos agudos, como volvo e hérnia interna, depois lise das bandas de Ladd, apendicectomia profilática e reposicionamento das alças sem fixação. ${ }^{3}$ Desde a década de 1990, a laparoscopia vem ganhando espaço na abordagem cirúrgica, ficando a laparotomia mais restrita a pacientes instáveis ou recém-nascidos. ${ }^{4}$ Entre as técnicas laparoscópicas, há duas: multiport e single-incision. Esta tem se tornado cada vez mais comum no campo cirúrgico; entretanto, ainda são necessários mais estudos randomizados comparando ambas as abordagens. ${ }^{7}$

Neste caso, foi realizada laparoscopia multiport com sucesso. O paciente recebeu alta quatro dias depois da cirurgia. Mesmo 8 meses após a cirurgia, o paciente continuou o tratamento medicamentoso, pois desenvolveu sérias consequências, como esofagite erosiva grau B de Los Angeles, hérnia hiatal (3 $\mathrm{cm}$ do pinçamento diafragmático), esôfago de Barrett e pangastrite enantematosa moderada, porém os sintomas são leves e houve melhora significativa na qualidade de vida do paciente.
Conclui-se que a má rotação intestinal em adultos consiste em um difícil diagnóstico em virtude da sua baixa prevalência e dos sintomas inespecíficos. Os exames para o diagnóstico consistem em tomografia computadorizada ou estudo radiográfico contrastado do trato gastrintestinal alto. $\mathrm{O}$ procedimento de Ladd laparoscópico continua sendo uma excelente técnica cirúrgica para os casos de má rotação sem complicações agudas.

\section{REFERÊNCIAS}

1. Araujo URMF, El Tawil II. Má rotação intestinal em adulto: relato de caso. ABCD Arq Bras Cir Dig. 2011;24(1):86-8. https://doi.org/10.1590/S0102-67202011000100019

2. Barros CEM, Vicenzi E, Espírito Santo GA, Santos NA. Banda de Ladd: relato de caso [Internet]. Cuiabá: Universidade Federal de Mato Grosso; 1998 [acesso em 20 out. 2015]. Disponível em: http://www.angelfire. com/me/Gastrocentro/ladd.html

3. Edling P, Tottrup AM. [Congenite intestinal malrotation as cause of intermittent abdominal pain]. Ugeskr Laeger. 2014;176(25A):pii:V01130062.

4. Moore KL, Dalley AF, Agur AMR. Anatomia: orientada para clínica. $7^{\mathrm{a}}$ ed. Rio de Janeiro: Guanabara Koogan; 2014. p.179-326.

5. Nakajima Y, Sakata H, Yamaguchi T, Yoshie N, Yamada T, Osako T, et al. Successful treatment of a 14-year-old patient with intestinal malrotation with laparoscopic Ladd procedure: case report and literature review. World J Emerg Surg. 2013;8(1):19. https://doi.org/10.1186/1749-7922-8-19

6. Vassaur J, Vassaur H, Buckley FP 3rd. Singleincision laparoscopic Ladd's procedure for intestinal malrotation. JSLS. 2014;18(1):132-5. https://doi. org/10.4293/108680813X13654754534954

7. Zheng ZH, Huang JL, Wei HB, Liu JP, Huang Y. Adult congenital intestinal malrotation accompanied by midgut volvulus: report of eight cases. Int J Clin Exp Med. 2014;7(6):1614-8.

Como citar este artigo:

Aragão GF, Guedes Filho RS, Colares JHF, Feijó JL, Oliveira MLF, Oliveira YIS. Má rotação intestinal na adolescência e suas consequências no diagnóstico tardio: relato de caso. Rev Fac Ciênc Méd Sorocaba. 2018;20(3):200-2. http://doi.org/10.23925/1984-4840.2018v20i3a12 